\title{
Analysis of the alcohol drinking behavior and influencing factors among emerging adults and young adults: a cross-sectional study in Wuhan, China
}

Wanrong Lư ${ }^{1}$, Jingdong $\mathrm{Xu}^{2}$, Anne Winifred Taylor ${ }^{3}$, Bridgette Maree Bewick ${ }^{4}$, Zhen Fu' ${ }^{1}$ Nanjin Wu${ }^{1}$, Ling Qian ${ }^{5^{*}}$ and Ping Yin ${ }^{1 *}$ (D)

\begin{abstract}
Background: The relationship between alcohol use in adolescents and young adults and outcomes has not been widely researched in China. The aim of the current study was to understand the current status of drinking behavior of Chinese youth transitioning into adulthood.

Methods: The cross-sectional study included 1634 participants between 18 and 34 years of age. The participants were randomly chosen from 13 administrative districts in Wuhan, and invited to complete a questionnaire. Univariate analysis was performed to describe the demographic distribution of alcohol consumption and the association with drinking status. Stepwise Logistic regression analysis was undertaken analyzing the factors influencing the drinking behaviors. The data were weighted to the population in Wuhan and analyzed using SAS version 9.3.

Results: For our sample of emerging and young Chinese adults the prevalence of drinking alcohol was $45.84 \%$. The non-drinkers predominated, accounting for $54.16 \%$ and light drinkers accounted for $42.94 \%$, while moderate and heavy drinkers were in the minority (2.90\%). The earlier the age of first alcohol drinking or the age of first being intoxicated, the greater the likelihood of being a moderate or heavy drinker. People with high emerging adulthood were more likely to have moderate or heavy drinking behaviors. The logistic regression analysis indicated that heavy drinkers were more likely to not be married and to be classified as high emerging adulthood.

Conclusions: Our findings suggested that the drinking pattern should be further evaluated over time to explore the ways in which social and cultural factors shape the drinking route of this age group. Effective drinking behavior prevention and interventions and appropriate guidance should be formulated to establish an appropriate attitude towards drinking alcohol and develop a drinking behavior which is conducive to physical and mental health between this particular demographic.
\end{abstract}

Keywords: Alcohol, Drinking behavior, Influencing factors, Adolescent, Transition, Emerging adulthood, Wuhan, Emerging adults

\footnotetext{
* Correspondence: qianlingzh@126.com; pingyin2000@126.com

${ }^{5}$ Chinese Center for Health Education, PR Ministry of Health, Building 12,

Block 1, Anhua Xili, Anwai Dajie, Chaoyang District, Beijing 100011, China

${ }^{1}$ Department of Epidemiology and Biostatistics, School of Public Health,

Tongji Medical College, Huazhong University of Science and Technology, 13

Hangkong Road, Wuhan 430030, People's Republic of China

Full list of author information is available at the end of the article
}

(c) The Author(s). 2019 Open Access This article is distributed under the terms of the Creative Commons Attribution 4.0 International License (http://creativecommons.org/licenses/by/4.0/), which permits unrestricted use, distribution, and reproduction in any medium, provided you give appropriate credit to the original author(s) and the source, provide a link to the Creative Commons license, and indicate if changes were made. The Creative Commons Public Domain Dedication waiver (http://creativecommons.org/publicdomain/zero/1.0/) applies to the data made available in this article, unless otherwise stated. 


\section{Background}

According to the World Health Organisation's 2014 estimate, $5.1 \%$ of the global disease and injury burden can be attributed to drinking alcohol [1]. In recent years, the analysis of international trends and investigations into the determinants of substance use has revealed alcohol consumption as one of the substances typically used and abused by adolescents and young adults [2-4]. Heavy drinking has largely been a phenomenon of emerging and young adulthood [5, 6], which places individuals at risk for adverse health and social effects [7-9]. It is believed this vulnerability is in part due to the dramatic life changes in relationships, employment, accommodation and roles of the individual as they transition into adulthood $[10,11]$. Harmful drinking behaviors in young years have been associated with greater risk of severe psychiatric and other drinking problems in adults [12]. Reducing high risk drinking patterns remain a major public health challenge [13].

In China, drinking alcohol is a widely accepted cultural tradition, especially during rituals, festivals, social gatherings, commercial occasions and special activities [14]. Based on the estimates of the Chinese National Bureau of Statistics, alcohol production and consumption in China has seen a sharp growth in recent decades $[15,16]$. The acceleration in growth is the highest internationally [17]. There is an inseparable relationship between alcohol consumption growth and the Chinese cultural context $[18,19]$.

Compared with other countries, early research on alcohol in China focused on the etiology of adults, mainly containing the physiological and clinical analysis. In recent years, investigations have begun to pay closer attention to alcohol-related health harm (e.g. mortality, chronic disease, cancer) [17, 20-22] and social problems (e.g. drunk driving, drunk crime, injuries) [23-25]. A local and national literature examining drinking behaviors and drinking motivations associated with alcohol consumption in China is emerging [26-28]. These studies have chiefly focused on adults, even the studies investigating younger drinkers have concentrated on adolescent or student populations $[29,30]$. There remains a relative paucity of studies on drinking prevalence, drinking behaviors and its related factors in China [31]. It is extremely rare for alcohol studies to concentrate attention on emerging and young adults groups in China. Accordingly, our research is of significance as the first attempt to explore the current status of drinking behaviors of emerging and young adults under the specific social and cultural background in China.

Within the literature no one age is identified as the exact point of transition into adulthood. Within the literature there is broad agreement that the point of transition occurs somewhere between late adolescence and the age of more than 30 [32-34]. Given this background the current study defined emerging and young adulthood as being between 18 to 34 years old. The outcomes of the current study will inform the development of prevention and early-intervention programs for emerging and young adults in China.

\section{Methods}

The data were derived from a trans-national populationbased survey covering four cities (Moscow, Ilorin, Wuhan, Montevideo) in four countries. In the current paper, the Wuhan data was used to investigate the drinking behavior among emerging adults and young adults (18-34 years old) under a specific social and cultural background in China. The method part refers to the published papers by Anne and Bridgette [35].

\section{Ethics approval and consent to participate}

This study received ethical review and approval from the Hubei Provincial CDC (Hubei Provincial Society for Health Promotion and Cigarette-smoking Control, HBPHP \& CCS-2014-01). Before further investigation, the interviewers conducted household confirmation, informed consent, and investigation management issues. The interviewers must introduce the content and purpose of the survey according to specific introductions and obtain verbal informed consent from all participants. Participants could terminate the interview at any time, or choose not to answer any question. Confidentiality of information was assured. Approval for the research was obtained by community leaders, appropriate government officials and other important related personnel. Households with alcohol and substance abuse could be referred to recognized hospitals/caregivers for proper management.

\section{Data source and participants}

Wuhan is the capital of Hubei Province, and at the time of the survey it had a population of approximately 9.8 million and a geographical area of about $8467 \mathrm{~km}^{2}$. Wuhan was pragmatically selected as a research site based on its diversity. Wuhan is divided into 13 administrative regions, and the multi-stage random sampling was performed across each region. The sample size of each administrative district was proportional to the population size. Communities were randomly selected in each administrative district, followed by random household selection. In each randomly selected household, the person with the most recent birthday, aged between 18 and 34 years, who lived in Wuhan for at least six months, was eligible and was invited to participate in the study. Prior to the main survey, 33 pilot interviews were conducted. The pilot assessed participants' understanding of the questions and length of the interview. 
Data collection lasted for 186 days (2014.10.252015.04.29). Door-knocking interviews were conducted in Chinese and the Wuhan local dialect by the interviewers who were local college students with specialized training. The data was obtained through face-to-face interviews, which is better for mutual understanding between interviewee and interviewer. Interviewers read out the questions and recorded via paper-and-pencil. Interviews were conducted in an environment where respondents felt most comfortable and where their privacy was respected. The average interview time was $15 \mathrm{~min}$. In total 1675 individuals were approached and 1675 participants agreed to be interviewed. After screening 1646 were identified as eligible to be interviewed. Of these 1642 interviews were completed. Two interviews were excluded due to insufficient completion of demographic information; demographic information is necessary for weighting. A further six cases were excluded due to incomplete drinking information. A total of 1634 questionnaires were included in the current analysis. The response rate was therefore $99.3 \%$.

\section{Drinking questionnaire}

Main alcohol questions included: (1) Have you ever consumed alcohol (excluding sips). (2) How old were you the first time you had a drink of an alcoholic beverage (excluding sips)? (3) How old were you the first time you got drunk? (4) During the past 12 months, how often did you drink beer, wine, spirits (e.g. vodka, gin, whisky, brandy), huangjiu or any other alcoholic beverage, even in small amounts? (5) During the past 12 months, how many alcoholic drinks did you have on a typical day when you drank alcohol? (6) In the past 12 months, how often have you ever gotten drunk? (Drunk refers to walking unsteadily, blurred vision, slurred speech, nausea, vomiting or any other symptoms)? (7) Drinking alcohol may impose various effects on people. When you are drinking, on what extent of the following statement may fit your situation (Always, usually, sometimes, seldom, never)? The effects including positive and negative effects, such as feel happy, feel relaxed, become aggressive toward other people, and so on.

Overall quantity-frequency (i.e. usual frequency of drinking by usual number of drinks consumed per drinking occasion) was calculated by multiplying the responses to the above two questions (how much and how many) with 25 or more drinks (coded as 25), 19-24 drinks (coded as 21.5), 16-18 drinks (coded as 17), 1215 drinks (coded as 13.5), 9-11 drinks (coded as 10), 78 drinks (coded as 7.5), 5-6 drinks (coded as 5.5), 3-4 drinks (coded as 3.5), 2 drinks (coded as 2), 1 drink (coded as 1) and less than 1 full drink (coded as 0.5). The annual number of drinks was calculated by multiplying the responses to the question on how many days did you drink alcohol with the response from how many drinks did you have. The variables were recorded into four drinking status groups: 0 drinks $=$ Abstainers; $>0$ but less than 365 drinks/year = Light drinkers; 365-729 drinks/year $=$ Moderate drinkers; 730 or more drinks $/$ year $=$ Heavy drinkers. No distinction was made between lifetime abstainers and former drinkers with alcohol consumption assessed during the past 12 months only. These four groups were collapsed into two groups of non-drinkers and current drinkers. 'Non-drinkers' referred to those who have never drunk before ('Lifetime abstainers') and those who have not drunk in the past 12 months ('Former drinkers'). 'Current drinkers' referred to those who have either drunk more than half a cup of alcohol at least once in the past 12 months or those who have drunk any quantity of alcohol at least twice in the past 12 months.

Pure alcohol intake (grams per day) was calculated by multiplying the responses to questions about how often and how much - and then the units were converted (the calculation was made according to the standard glass of specific alcoholic beverages, $1 \mathrm{ml}$ alcohol $=0.789 \mathrm{~g}$ ). Respondents were provided with visual references to beverage specific drink sizes in order to facilitate reporting of number of drinks in standard sizes.

The interview also included an assessment of emerging adulthood which included the following three statements: a) I have reached adulthood. b) I am financially independent of my parents. c) I am emotionally independent of my parents. Questions were answered on a five-point Likert scale: $5=$ strongly agree; $4=$ agree; $3=$ neither agree nor disagree; 2 = disagree; $1=$ strongly disagree. Responses were totaled with 3-11 points indicating low emerging adulthood, 12-13 points indicating medium emerging adulthood, and 14-15 points indicating high emerging adulthood. The emerging adulthood statements were based on the work of Arnett and Padilla-Walker [36] and adapted to fit common assumptions within China. Scores were reversed so that lower emerging adulthood scores indicated lower emerging adulthood.

\section{Statistical analysis}

In this study, the data were ranked weighted as per the stratified target population information provided by Hubei Provincial Institute for Health Education in 2014 by Administrative District, age, gender and selection probability. The weight value distribution range was $0.285-4.300$. The aim was to minimize bias and ensure that samples were representative; the weighted demographics of the sample were consistent with the local demographics.

The data base was built with EpiData version 3.1, and the logical verification and statistical analysis of the data were carried out using the 9.3 version of the SAS statistical analysis package. Demographic characteristics included age, 
sex, marital status, education level, current student status and employment status. Group comparison of categorical data was using $\mathrm{CMH}-\chi^{2}$ test. The rank test was used for group comparison of grade data. Simple univariate analysis was performed to describe the demographic distribution of alcohol consumption and the association analysis of drinking status. Stepwise Logistic regression analysis was undertaken analyzing the factors influencing the drinking status among the population between 18 and 34 years of age in Wuhan, controlling for the socio-demographic characteristics and adult tendency. In addition, the relationship between emerging adulthood and drinking behavior was assessed. The significance was set at $p<0.05$.

\section{Results}

The demographic and emerging adulthood distribution of alcohol consumption

A total of 1634 people were included in the current analysis. Of these 749 (45.8\%) were current drinkers. Chisquare statistics were calculated to assess the drinking distribution difference between different demographic characteristics and emerging adulthood (Table 1). Significant differences were observed in the drinking distribution between different marital status, current student status and emerging adulthood. The proportion of married people drinking was lower than those not married $\left(\chi^{2}=\right.$ 10.14, $p=0.0015)$; Students had a higher proportion of drinking than non-students $\left(\chi^{2}=5.09, p=0.0240\right)$; People with high emerging adulthood had a higher proportion of drinking $\left(\chi^{2}=10.09, p=0.0064\right)$. Figure 1 displays the current drinking proportion for the three age groups. Although not significant, younger females (18-24 years) were more likely to drink than males.

\section{Drinking behavior}

Participants were divided into four categories: non-drinkers, light drinkers, moderate drinkers and heavy drinkers. The non-drinkers predominated, accounting for $54.2 \%$ of the population, followed by light drinkers (42.9\%), moderate drinkers (1.7\%) and heavy drinkers (1.2\%). The drinking status between different demographic characteristics and emerging adulthood are shown in Table 2. It revealed a statistically significant difference between drinking status and marital status, current student status, as well as emerging adulthood. Non-students had a higher proportion of moderate or heavy drinkers $\left(\chi^{2}=4.24, p=0.0395\right)$; People who were not married had a higher proportion of heavy drinkers $\left(\chi^{2}=7.22, p=0.0072\right)$; People with high emerging adulthood had a higher proportion of heavy drinkers $\left(x^{2}=7.36, p=\right.$ 0.0067).

The results of the rank test (Table 3) indicated that males with an early first drinking age $(\mathrm{z}=2.49, p=$ $0.0129)$ or a first drunk age $(\mathrm{z}=2.46, p=0.0138)$ before 15 years of age, had a higher proportion of moderate or heavy drinkers than that after the age of 15 ; No significant relationship was observed for females (small cell sizes). Overall, the earlier the first drinking age, the higher the level of current drinking $(\mathrm{z}=2.57, p=0.0101)$. When exploring the relationship between drunkenness and negative effects among current drinkers, the incidence of intoxication was $33.6 \%$ for those who linked drinking with negative effects, while for people who did not consider drinking with negative effects, the incidence of intoxication was $18.7 \%$, a difference of $15.0 \%$ (95\% CI (0.081-0.205), $p<0.0001)$.

\section{Drinking behaviors and emerging adulthood}

Stepwise regression methodology was used to determine the model for demographic characteristics and drinking status; age, marital status and emerging adulthood were positively related to drinking status (Table 4$)$. There was a significant difference in marital status $(p<0.0001)$ and higher emerging adulthood $(p=0.0030)$, with heavy

Table 1 The demographic and emerging adulthood distribution of alcohol consumption

\begin{tabular}{|c|c|c|c|c|c|c|}
\hline \multirow[t]{2}{*}{ Variables } & \multicolumn{2}{|c|}{ Non-drinking } & \multicolumn{2}{|c|}{ Drinking } & \multirow[t]{2}{*}{$x^{2}$} & \multirow[t]{2}{*}{$p$} \\
\hline & $n$ & $\%(95 \% \mathrm{Cl})$ & $n$ & $\%(95 \% \mathrm{Cl})$ & & \\
\hline Marital status & & & & & 10.14 & 0.0015 \\
\hline Married & 424 & $58.6(54.9-62.1)$ & 300 & $41.4(37.9-45.1)$ & & \\
\hline Not married & 461 & $50.7(47.4-53.9)$ & 449 & $49.3(46.1-52.6)$ & & \\
\hline Current student status & & & & & 5.09 & 0.0240 \\
\hline Yes & 161 & $48.6(43.3-54.0)$ & 170 & $51.4(46.0-56.7)$ & & \\
\hline No & 724 & $55.6(52.9-58.2)$ & 579 & $44.4(41.8-47.1)$ & & \\
\hline Emerging adulthood & & & & & 10.09 & 0.0064 \\
\hline Low & 219 & $58.4(53.4-63.3)$ & 156 & $41.6(36.7-46.6)$ & & \\
\hline Medium & 218 & $49.1(44.5-53.7)$ & 226 & $50.9(46.3-55.5)$ & & \\
\hline High & 343 & $48.9(45.2-52.6)$ & 359 & $51.1(47.4-54.8)$ & & \\
\hline
\end{tabular}

Legend: $95 \% \mathrm{Cl}$ was calculated by Newcombe-wilson method 


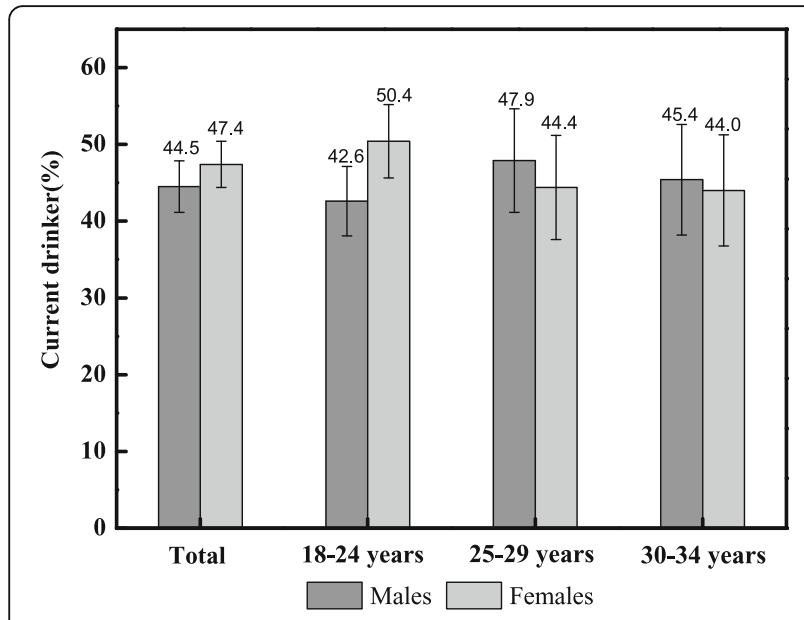

Fig. 1 The proportion of current alcohol drinkers by age group

drinkers more likely to be people not married, and with higher emerging adulthood.

Figure 2, (a) demonstrates the daily average alcohol intake and the highest alcohol intake significantly increased as emerging adulthood scores increased. The result of rank correlation analysis showed the correlation coefficient of emerging adulthood and the alcohol intake (grams per day) was $0.56(p=0.0897)$. The correlation coefficient of emerging adulthood and the highest alcohol intake (grams per time) was $0.76(p=0.0111)$. Similarly, in the Fig. 2 (b), drinking frequency, the highest drinking frequency and the frequency of being drunk significantly decreased as adult tendency scores decreased. The correlation coefficient of emerging adulthood and the drinking frequency was $0.70(p=0.0165)$. The correlation coefficient of emerging adulthood and the highest drinking frequency was $0.52(p=0.1276)$. The correlation coefficient of emerging adulthood and frequency of being drunk was $0.52(p=0.1221)$. Figure 2, (c) showing that the alcohol consumption of beer was significantly higher than other types of alcoholic beverages, followed by white spirits, wine and huangjiu. The annual alcohol consumption of four types of alcoholic beverages (beer, wine, white spirits and huangjiu) showed a upward trend with the increasing emerging adulthood scores.

\section{Discussion}

This is the first study aimed at evaluating the drinking behaviors and its related factors among emerging and young adults in China. The current study suggests that the majority of people aged 18-34 years living in Wuhan who consume alcohol could be considered 'light drinkers'. Although the incidence of heavy drinking is not high among this population when extrapolated to the number of individuals across China, this represents a substantial challenge for public health. In addition, we cannot predict whether light or moderate drinkers will engage in dangerous drinking behaviors in the future. Public health prevention and intervention efforts in China should strengthen and develop an appropriate attitude towards drinking alcohol. There is also a need to develop effective interventions for the relatively few, but substantial in number, heavier drinkers in order to minimize the costs associated with problematic drinking behavior.

The influence of gender on drinking behavior has been demonstrated in many studies. Some alcohol studies reported that males were more likely to consume alcohol than females, and females were more likely to be abstainers [37-39]. In our gender analysis, females had a slightly higher current drinking proportion than males ( $2.82 \%$ higher). This finding may be related to recent changes in economic and social status resulting in females developing a stronger independent consciousness, increasing levels of employment, and increasing social

Table 2 Difference analysis of drinking status

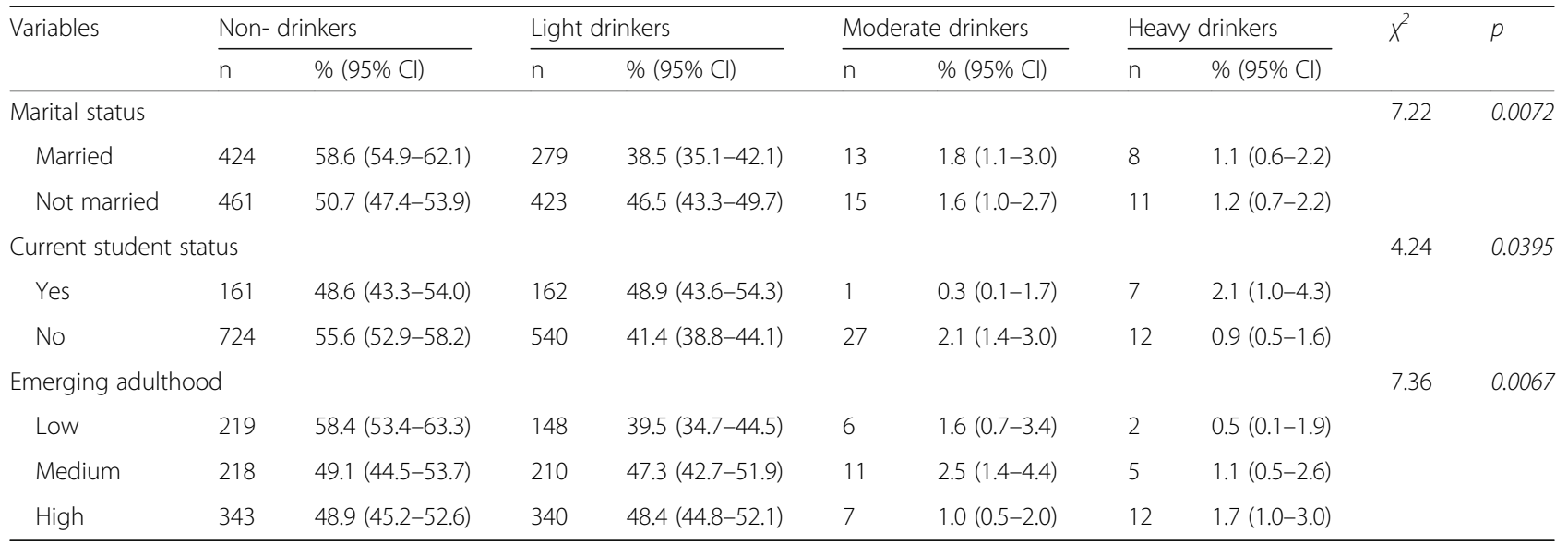

Legend: $95 \% \mathrm{Cl}$ was calculated by Newcombe-wilson method 
Table 3 The relationship between the first drinking/drunk age and current drinking status

\begin{tabular}{|c|c|c|c|c|c|c|c|c|}
\hline & Age at the & inking & $z$ & $p$ & Age at th & drunk & $z$ & $p$ \\
\hline & $\overline{\leq 14}$ & $\geq 15$ & & & $\leq 14$ & $\geq 15$ & & \\
\hline Male & & & 2.49 & 0.0129 & & & 2.46 & 0.0138 \\
\hline Light drinkers & $15(78.9)$ & $299(94.0)$ & & & $4(57.1)$ & $143(88.8)$ & & \\
\hline Moderate drinkers & $3(15.8)$ & $11(3.5)$ & & & $2(28.6)$ & $12(7.5)$ & & \\
\hline Heavy drinkers & $1(5.3)$ & $8(2.5)$ & & & $1(14.3)$ & $6(3.7)$ & & \\
\hline Total & 19 & 318 & & & 7 & 161 & & \\
\hline Female & & & -0.68 & 0.4972 & & & -0.60 & 0.5470 \\
\hline Light drinkers & $7(100.0)$ & $313(93.7)$ & & & $3(100.0)$ & $117(88.6)$ & & \\
\hline Moderate drinkers & $0(0.0)$ & $12(3.6)$ & & & $0(0.0)$ & $8(6.1)$ & & \\
\hline Heavy drinkers & $0(0.0)$ & $9(2.7)$ & & & $0(0.0)$ & $7(5.3)$ & & \\
\hline Total & 7 & 334 & & & 4 & 132 & & \\
\hline Overall & & & 2.57 & 0.0101 & & & 1.77 & 0.0765 \\
\hline Light drinkers & $21(80.8)$ & $612(93.9)$ & & & $7(70.0)$ & $260(88.7)$ & & \\
\hline Moderate drinkers & $4(15.4)$ & $23(3.5)$ & & & $2(20.0)$ & $20(6.8)$ & & \\
\hline Heavy drinkers & $1(3.9)$ & $17(2.6)$ & & & $1(10.0)$ & $13(4.5)$ & & \\
\hline Total & 26 & 652 & & & 10 & 293 & & \\
\hline
\end{tabular}

activities and communication opportunities. Other studies have highlighted that male excesses are no longer apparent along with the appearance of "narrowing of the gender gap" in particular time periods, geographical settings, age groups and drinking patterns [40-43]. Consistent with the findings that males had higher drinking frequency and exceeded females in rates of heavy drinking behavior in other studies, we found males had a higher proportion of moderate or heavy drinkers [17]. In addition, the results revealed students were more likely to report being consumers of alcohol, this is consistent with surveys from other countries $[44,45]$.

Table 4 Adjusted odds ratios (OR) for drinking status

\begin{tabular}{llllll}
\hline Variables & $\beta$ & $S E$ & $O R$ & $95 \% \mathrm{Cl}$ & $p$ \\
\hline Intercept1 & -4.3410 & 0.2320 & & & $<0.0001$ \\
Intercept2 & -3.4131 & 0.1504 & & & $<0.0001$ \\
Intercept3 & -0.0695 & 0.0605 & & & 0.2508 \\
Age & & & & & \\
18-24 & & & 1.000 & & \\
25-29 & 0.1492 & 0.0831 & 1.545 & $(1.137-2.101)$ & 0.0724 \\
30-34 & 0.1368 & 0.0966 & 1.526 & $(1.076-2.166)$ & 0.1568 \\
Marital status & & & & & \\
Married & & & 1.000 & & \\
Not married & 0.3016 & 0.0758 & 1.828 & $(1.358-2.461)$ & $<0.0001$ \\
Emerging adulthood & & & & \\
Low & & & 1.000 & & 0.1192 \\
Medium & 0.2004 & 0.0676 & 1.039 & $(0.779-1.385)$ & \\
High & 0.1192 & 0.0765 & 1.325 & $(1.021-1.721)$ & 0.0030 \\
\hline
\end{tabular}

Legend: Adjusted by age, marital status and emerging adulthood
Previous studies have noted a first drinking age of 15 years or earlier was at elevated risk for alcohol abuse and dependence [46, 47]. The earlier the first drinking age, the probability of subsequently developing heavy drinking patterns and resultant health damage was markedly increased $[48,49]$. Our results also indicated that, whether male or female, a first drinking age before 15 had a greater potential risk of engaging in heavy drinking when compared to people who started later than 15 . Those who started drinking before 15 years of age were also more likely to be moderate or heavy drinkers. Publish research confirms that age of reporting first being drunk is related to the negative effects of drinking [50]. Early-onset drunkenness is associated with an increased risk of developing alcohol use disorder and signaled excess mortality risk [51]. Consistent with these earlier reports, our results showed that the younger the age of first drunkenness, the greater the likelihood of becoming a moderate or heavy drinker. Those who had ever been intoxicated were more likely to associate drinking with negative effects. Premature drinkers or premature drunks have been found to be more impulsive and adventurous, more extreme and poorly controlled, and report the occurrence of health problems and other risks in later life $[47,52,53]$.

The current results concerning emerging adulthood found an association between emerging adulthood and drinking behaviors. Heavy drinkers were more likely to be people with higher emerging adulthood. Furthermore, people with higher emerging adulthood usually had more extreme drinking alcohol behavior with higher frequency. Previous studies suggested that young adults reduce risk drinking behavior to more moderate levels of 


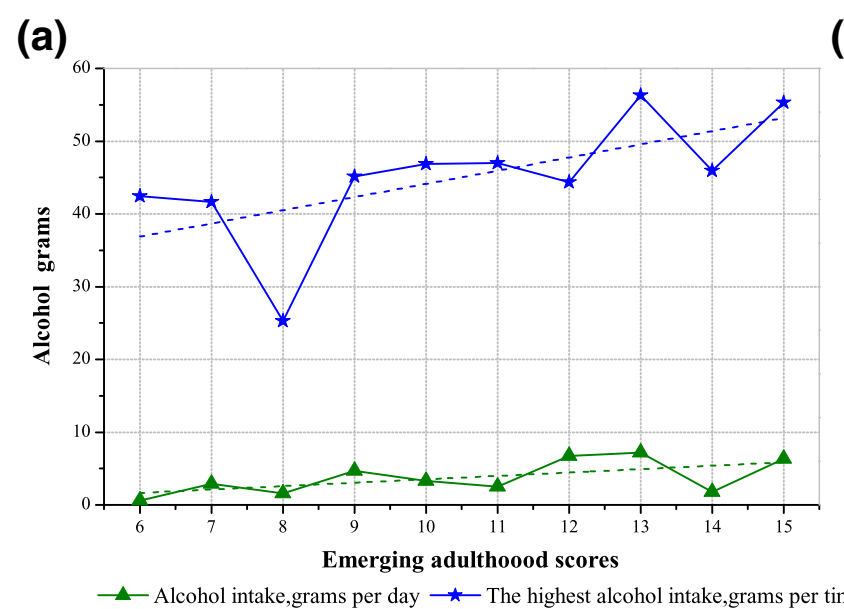

(b)

(c)
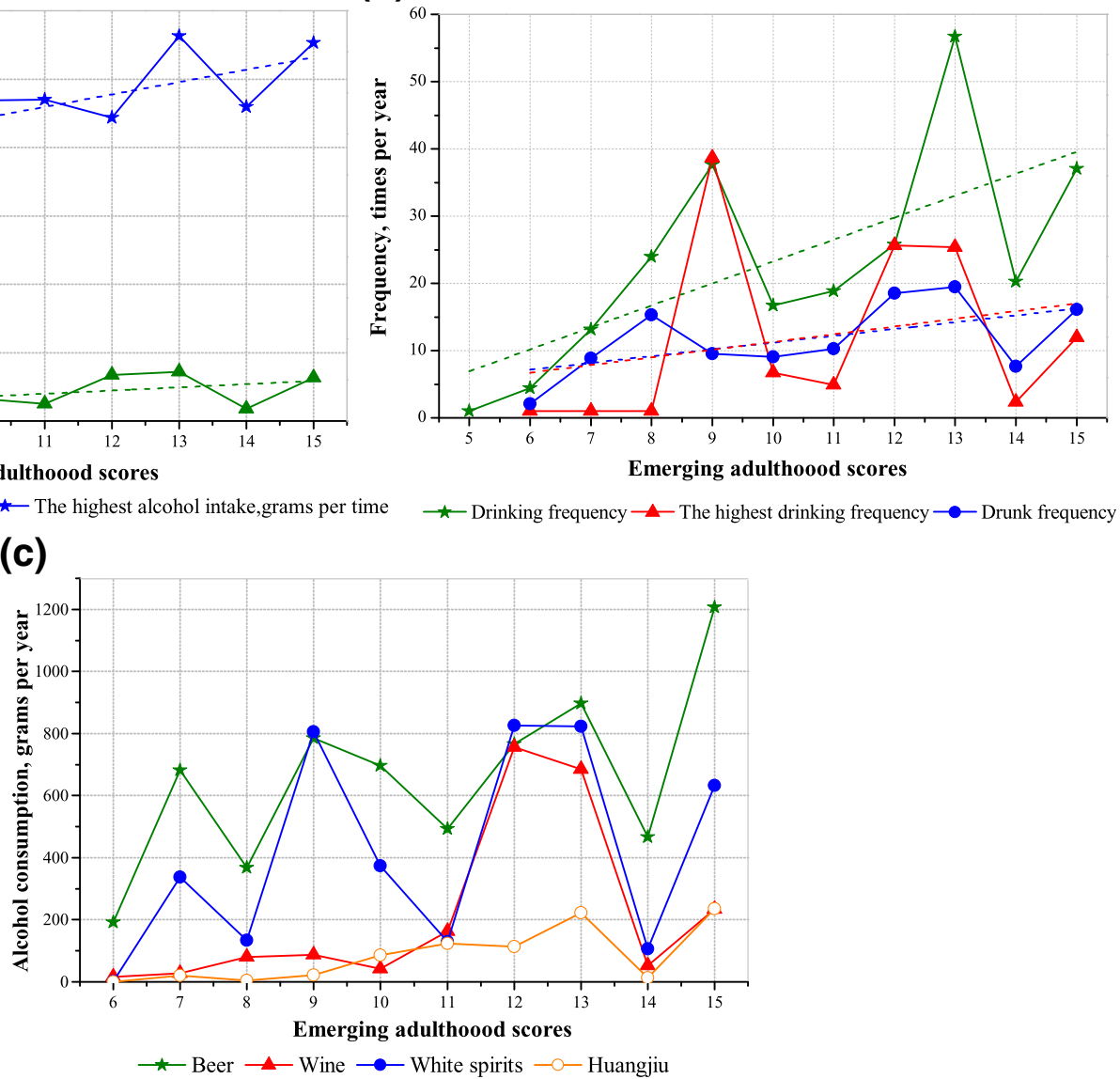

Fig. 2 The relationship between alcohol grams (a), drinking/drunk frequency (b), and four alcoholic beverages' consumption (c) with emerging adulthood scores (For current drinkers)

alcohol use when adult roles and responsibilities are taken on [54-56]. Our results suggested an increase of alcohol use in those with higher emerging adulthood. This apparent contradiction between our study and the published literature may be the result of differences in culture between China and other countries. As emerging adults mature, they encounter more responsibility and play new roles. For current Chinese drinkers it can be difficult to avoid consuming alcohol, especially during social activities where there is a felt pressure to drink [57]. Greater social pressure and more drinking occasions can be related to trends in drinking motives, drinking opportunities and drinking behaviors.

Our research also indicated a link between the alcohol consumption of the four types of alcoholic beverages (beer, wine, white spirits and huangjiu) and emerging adulthood scores, which have shown that among the four most common types of alcoholic beverages in China, the annual alcohol consumption of beer was significantly highest, which supported the fact that China is one of the largest beer consuming countries in the world [58].
Study limitations include the fact that the study was cross-sectional, which restricts our assessments of the temporal relationships of the associations. The data was limited to one city and the results were not nationally representative. Concerns around the length of the structured interview and lack of availability of a validated brief assessment of 'emerging adulthood' meant the current study created a measure based on the work of Arnett and Padilla-Walker [36]. The study would have been strengthened had a standardized, validated, brief-assessment of emerging adulthood been available. Our definition of a heavy drinker precludes those who drink very occasionally but who on these occasions drink very heavily. Future research should aim to understand this important group of individuals. Study strengths include focusing on emerging adults and young adults aged between 18 to 34 years old, a large and targeted sample is conducive to formulating effective prevention and intervention. Probability-based random sampling methodology (stratified, clustered, systematic) was adopted to ensure the random selection of population. The data were weighted, 
ensuring findings were representative of the broader urban population. In addition, this self-report method can incorporate all alcohol consumption levels under the circumstance that many official statistics often do not record the alcohol consumption.

\section{Conclusions}

The research focused on the alcohol drinking behaviors of this particular age group, and the findings of the present study strongly supported the significant impact of social and cultural background and age-specific characteristics on the drinking behaviors. For China, China-specific influences together with assessment of adulthood level are important considerations when developing effective drinking interventions for emerging and young adults. Internationally, this country-specific study increases the understanding of Chinese drinking characteristics and provides valuable reference for international alcohol-related surveys.

\section{Abbreviations}

HBPHP \& CCS: Hubei Provincial Society for Health Promotion and Cigarettesmoking Control

\section{Acknowledgements}

The authors wish to thank the International Alliance for Responsible Drinking (IARD), the National Science Foundation of China (No. 81573262) and the Fundamental Research Funds for the Central Universities, HUST (No. 2016YXZD042) for providing support towards the study. And we express sincerely gratitude to thank Professor Ping Yin, Ling Qian, Alfred Bamiso Makanjuola and Valentina Vasilievna Kirzhanova for their contributions. The authors also would like to thank all research participants for their generous assistance and cooperation.

\section{Funding}

Including travel and reasonable expenses, this work was funded by the International Alliance for Responsible Drinking (IARD), the National Science Foundation of China (No. 81573262). A not-for-profit organization supported by major producers of alcoholic beverages (http://iard.org). This work was also supported by the China Health Education Center and 'the Fundamental Research Funds for the Central Universities', HUST Department of Epidemiology and Health Statistics, School of Public Health Tongji Medical College (No. 2016YXZD042). The funders had no role in the analysis, data interpretation and writing of this manuscript. The corresponding author was the only one to have full access to all the data and had final responsibility for the decision to submit for publication. The work product, findings, viewpoints, and conclusions presented here are solely those of the named authors and do not necessarily represent those of IARD or its sponsoring companies.

\section{Availability of data and materials}

We did not get consent to share data obtained from the questionnaire and key informant interviews. The datasets used and/or analysed during the current study are available from the corresponding author on reasonable request.

\section{Authors' contributions}

All authors contributed to the study. PY, LQ, AWT and BMB conceptualized and designed the study, and supervised the survey. PY, LQ, JX carried out the questionnaire survey. WL performed the statistical analyses and wrote an initial draft of the paper. ZF and NW performed data and statistical analysis results checking. PY, LQ, AWT, and BMB supervised and guided the writing of the manuscript. All authors have provided comments on drafts and contributed to the writing of the manuscript. All authors have read and approved the final manuscript.

\section{Ethics approval and consent to participate}

From the Hubei Provincial CDC (Hubei Provincial Society for Health Promotion \& Cigarette-smoking Control, HBPHP \& CCS-2014-01), this study received ethical review and approval, ethics committee approval for verbal consent was also included. The interviewers must introduce the content and purpose of the survey according to specific introductions. For all the participants, the verbal informed consent to participate in the study was obtained before the interview started. The abbreviation of the content is as follows: the interviewer's name and working institute, the purpose of this investigation and the randomly selected of the participants for inclusion in our study, the main information will be collected and the time will take to complete the survey. Finally tell the participants that his decision whether or not to participate in this research is completely voluntary. Should he decide to participate, his responses will be kept completely confidential. If any questions make him uncomfortable, we can skip them. He is also free to end the interview at any time. Then ask the participant if he will agree to participate in our study. All methods in the study were performed in accordance with relevant guidelines and regulations.

\section{Consent for publication}

Not applicable. (The current manuscript contains aggregated data only. No details, images, or videos relating to individuals participants are included in the current manuscript).

\section{Competing interests}

$\mathrm{BMB}$, as keynote speaker/expert adviser, has received reimbursement of travel expenses and/or time from Anheuser-Busch, Noctis, and the International Centre for Alcohol Policies. BMB has been in receipt of a research grant provided by the European Research Advisory Board (ERAB) and is currently a recipient of a research grant provided by the International Alliance for Responsible Drinking (IARD). BMB has received reimbursement of reasonable expenses incurred as a member of the independent Research Advisory Board for the European Foundation for Alcohol Research. These projects/activities did not influence the current work. The other authors declare that they have no competing interest other than those mentioned in the acknowledgment section.

\section{Publisher's Note}

Springer Nature remains neutral with regard to jurisdictional claims in published maps and institutional affiliations.

\section{Author details \\ 'Department of Epidemiology and Biostatistics, School of Public Health, Tongji Medical College, Huazhong University of Science and Technology, 13 Hangkong Road, Wuhan 430030, People's Republic of China. ${ }^{2}$ Hubei Institute for Health Education, Zhuodao Quan Road No.6, Hongshan District, Wuhan 430079, China. ${ }^{3}$ Population Research \& Outcome Studies, Discipline of Medicine, The University of Adelaide, SAHMRI, North Terrace, Adelaide 5006, Australia. ${ }^{4}$ Division of Psychological and Social Medicine, Leeds Institute of Health Sciences, School of Medicine, University of Leeds, Leeds LS2 9NL, UK. ${ }^{5}$ Chinese Center for Health Education, PR Ministry of Health, Building 12, Block 1, Anhua Xili, Anwai Dajie, Chaoyang District, Beijing 100011, China.}

Received: 15 June 2018 Accepted: 15 April 2019

Published online: 30 April 2019

References

1. Yoon S, Lam WW, Sham JT, Lam TH. Learning to drink: how Chinese adolescents make decisions about the consumption (or not) of alcohol. Int J Drug Policy. 2015;26(12):1231-7.

2. Gilvarry E. Substance abuse in young people. J Child Psychol Psychiatry. 2000;41(1):55-80.

3. Glantz MD, Leshner Al. Drug abuse and developmental psychopathology. Dev Psychopathol. 2000;12(4):795-814.

4. Steinhausen H, Eschmann S, Heimgartner A, Metzke CW. Frequency, course and correlates of alcohol use from adolescence to young adulthood in a Swiss community survey. BMC Psychiatry. 2008;8(1):5.

5. Substance Abuse and Mental Health Services Administration. Results from the 2010 National Survey on drug use and health: National Findings. Rockville, MD: Office of Applied Studies; 2011. HHS publication no. (SMA). p. 11-4658. 
6. Grant BF, Dawson DA, Stinson FS, Chou S, Dufour MC, Pickering RP. The 12month prevalence and trends in DSM-IV alcohol abuse and dependence: United States, 1991-1992 and 2001-2002. Drug Alcohol Depend. 2004;74: 223-34.

7. Hall WD, Patton G, Stockings E, Weier M, Lynskey M, Morley Kl, et al. Why young people's substance use matters for global health. Lancet Psychiatry. 2016;3(3):265-79.

8. Rehm J, Room R, Graham K, Monteiro M, Gmel G, Sempos CT. The relationship of average volume of alcohol consumption and patterns of drinking to burden of disease: an overview. Addiction. 2003;98(9):1209-28.

9. Gmel G, Rehm J. Harmful alcohol use. Alcohol Res Health. 2003;27(1):52-62.

10. Hall WD, Patton $G$, Stockings $E$, Weier $M$, et al. Why young people's substance use matters for global health. Lancet. 2016:3:265-79.

11. Schulenberg JE, Maggs JL. A developmental perspective on alcohol use and heavy drinking during adolescence and the transition to young adulthood. J Study Alcohol. 2002;14(Supp):54-70.

12. Bourque J, Baker TE, Dagher A, Evans AC, Garavan H, Leyton M, et al. Effects of delaying binge drinking on adolescent brain development: a longitudinal neuroimaging study. BMC Psychiatry. 2016;16(1):445.

13. Tang H, Cai W, Wang H, Zhang Q, Qian L, Shell DF, et al. The association between cultural orientation and drinking behaviors among university students in Wuhan, China. PLoS One. 2013;8(1):e54796.

14. Wang S, Newman IM, Shell DF. Cultural orientation and its associations with alcohol use by university students in China. PLoS One. 2016;11(11):e165858.

15. National Bureau of Statistics of China. Yearbook of Chinese statistics [Chinese] Beijing. China: Statistic Press; 1979

16. National Bureau of Statistics of China. Yearbook of Chinese statistics [Chinese] Beijing. China: Statistic Press; 2014

17. Tang YL, Xiang XJ, Wang XY, Cubells JF, Babor TF, Hao W. Alcohol and alcohol-related harm in China: policy changes needed. Bull World Health Organ. 2013;91(4):270-6.

18. Cochrane J, Chen $\mathrm{H}$, Conigrave KM, Hao W. Alcohol use in China. Alcohol Alcohol. 2003;38(6):537-42

19. Zhang J, Wang J, Lu Y, Qiu X, Fang Y. Alcohol abuse in a metropolitan city in China: a study of the prevalence and risk factors. Addiction. 2004;99: 1103-10.

20. Liang H, Wang J, Xiao H, Wang D, Wei W, Qiao Y, Boffetta P. Estimation of cancer incidence and mortality attributable to alcohol drinking in China. BMC Public Health. 2010;10:730.

21. Pan CS, Ju TR, Lee CC, Chen YP, Hsu CY, Hung DZ, Chen WK, Wang IK Alcohol use disorder tied to development of chronic kidney disease: a nationwide database analysis. PLoS One. 2018;13(9):e0203410.

22. Zhou S, Zhou R, Zhong T, Li R, Tan J, Zhou H. Association of smoking and alcohol drinking with dementia risk among elderly men in China. Curr Alzheimer Res. 2014;11(9):899-907.

23. Zhang G, Yau KK, Gong X. Traffic violations in Guangdong Province of China: speeding and drunk driving. Accident analysis and prevention. 2014;64:30-40.

24. Zhang DJ, Lin Y, Mao YJ, Hu ZQ. A study on characteristics of criminals with alcohol-related offence in forensic psychiatry. Fa Yi Xue Za Zhi. 2007;23(2):101-4

25. Cherpitel CJ, Ye Y, Bond J, Borges G, Chou P, Nilsen P, Ruan J, Xiang X. Multilevel analysis of alcohol-related injury and drinking patterns: emergency department data from 19 countries. Addiction. 2012;107:1263-72.

26. Li Y, Jiang $Y$, Zhang $M$, Yin P, Wu F, Zhao W. Drinking behavior among men and women in China: the 2007 China chronic disease and risk factor surveillance. Addiction. 2011;106:1946-56.

27. Cheng $H G$, Phillips MR, Zhang Y, Wang Z. Relationship of drinking motives with alcohol consumption and alcohol-related problems identified in a representative community-based study from Ningxia, China. Addict Behav. 2017;74:156-61.

28. Zhang Q, Zuo J, Pan H, Wang X, Zou S, Li X, Lu L, Nie S, Hu X, Ma G. Drinking behaviors of adults at different time of day in four cities of China in summer. Wei Sheng Yan Jiu. 2013;42(2):263-8.

29. Newman IM, Ding LY, Feng YH. Estimate of undergraduate university student alcohol use in China: a systematic review and meta-analysis. Arch Public Health. 2017:75:52

30. Feng $\mathrm{YH}$, Newman IM. Estimate of adolescent alcohol use in China: a metaanalysis. Arch Public Health. 2016;74:45.

31. Millwood IY, Li L, Smith M, et al. Alcohol consumption in 0.5 million people from 10 diverse regions of China: prevalence, patterns and socio-demographic and health-related correlates. Int J Epidemiol. 2013;42(3):816-27.
32. Lee MR, Chassin L, Villalta IK. Maturing out of alcohol involvement: transitions in latent drinking statuses from late adolescence to adulthood. Dev Psychopathol. 2013;25(4 Pt 1):1137-53.

33. Soucie KM, Jia F, Zhu N, Pratt MW. The codevelopment of community involvement and generative concern pathways in emerging and young adulthood. Dev Psychol. 2018;54(10):1971-6.

34. Pinho S, Padez C, Manco L. High AMY1 copy number protects against obesity in Portuguese young adults. Ann Hum Biol. 2018;10:1-5.

35. Vrana KE, Taylor AW, Bewick BM, Makanjuola AB, Qian L, Kirzhanova W, Alterwain P. Context and culture associated with alcohol use amongst youth in major urban cities: a cross-country population based survey. PLoS One. 2017;12(11):e0187812.

36. Arnett JJ, Padilla-Walker LM. Brief report. Danish emerging adults' conceptions of adulthood. J Adolesc. 2015;38:39-44.

37. Wilsnack RW, Wilsnack SC, Kristjanson AF, Vogeltanz-Holm ND, Gmel G. Gender and alcohol consumption:patterns from the multinational GENACIS project. Addiction. 2009;104(9):1487-500.

38. World Health Organization. Global status report on alcohol and health. Geneva: WHO: 2014

39. Wilsnack RW, Vogeltanz ND, Wilsnack SC, et al. Gender differences in alcoholconsumption and adverse drinking consequences: cross-cultural patterns. Addiction. 2000;95(2):251-65.

40. Erol A, Karpyak VM. Sex and gender-related differences in alcohol use and its consequences: contemporary knowledge and future research considerations. Drug Alcohol Depen. 2015;156:1-13.

41. Keyes KM, Grant BF, Hasin DS. Evidence for a closing gender gap in alcohol use, abuse, and dependence in the United States population. Drug Alcohol Depend. 2008;93(1-2):21-9.

42. Bratberg GH, Wilsnack SC, Wilsnack R, Haugland SH, Krokstad S, Sund ER, et al. Gender differences and gender convergence in alcohol use over the past three decades (1984-2008), the HUNT study, Norway. BMC Public Health. 2016;16(1).

43. McPherson M, Casswell S, Pledger M. Gender convergence in alcohol consumption and related problems: issues and outcomes from comparisons of New Zealand survey data. Addiction. 2004;99(6):738-48.

44. White A, Hingson R. The burden of alcohol use: excessive alcohol consumption and related consequences among college students. Alcohol Res. 2013;35(2):201-18.

45. Merrill JE, Carey KB. Drinking over the lifespan: focus on college ages. Alcohol Res. 2016;38(1):103-14.

46. Guttmannova K, Bailey JA, Hill KG, Lee JO, Hawkins JD, Woods ML, et al. Sensitive periods for adolescent alcohol use initiation: predicting the lifetime occurrence and chronicity of alcohol problems in adulthood. J Stud Alcohol Drugs. 2011;72(2):221-31.

47. Deutsch AR, Slutske WS, Richmond-Rakerd LS, Chernyavskiy P, Heath AC, Martin NG. Causal influence of age at first drink on alcohol involvement in adulthood and its moderation by familial context. J Stud Alcohol Drugs. 2013:74(5):703-13.

48. Grant BF. The impact of a family history of alcoholism on the relationship between age at onset of alcohol use and DSM-IV alcohol dependence: results from the National Longitudinal Alcohol Epidemiologic Survey. Alcohol Health Res World. 1998:22(2):144-7.

49. Hingson RW, Heeren T, Jamanka A, Howland J. Age of drinking onset and unintentional injury involvement after drinking. JAMA. 2000:284(12):1527-33.

50. Stamates AL, Lau-Barraco C, Linden-Carmichael AN. Alcohol expectancies mediate the relationship between age of first intoxication and drinking outcomes in college binge drinkers. Subst Use Misuse. 2016;51(5):598-607.

51. Hu H, Eaton WW, Anthony JC, Wu L, Cottler LB. Age of first drunkenness and risks for all-cause mortality: a 27-year follow-up from the epidemiologic catchment area study. Drug Alcohol Depen. 2017;176:148-53.

52. Newton-Howes G, Boden JM. Relation between age of first drinking and mental health and alcohol and drug disorders in adulthood: evidence from a 35-year cohort study. Addiction. 2016;111(4):637-44.

53. Morean ME, L'Insalata A, Butler ER, et al. Age at drinking onset, age at first intoxication, and delay to first intoxication: assessing the concurrent validity of measures of drinking initiation with alcohol use and related problems. Addict Behav. 2018;79:195-200.

54. Kuntsche E, Gmel G. Alcohol consumption in late adolescence and early adulthood - where is the problem? Swiss Med Wkly. 2013;143:w13826.

55. Rehm J, Room R, Monteiro MG, Gmel G, Graham K, Rehn N, et al. Alcohol use. In: Ezzati M, Lopez AD, Rodgers A, Murray CJL, editors. Comparative quantification of health risks global and regional burden of disease 
attributable to selected major risk factors. Geneva: World Health Organization (WHO); 2004. p. 959-1108.

56. Rehm J, Arminger G. Alcohol consumption in Switzerland 1987-93: adjusting for differential effect of assessment techniques on the analysis of trends. Addiction. 1996;91(9):1335-44.

57. Hao W, Young D. Drinking patterns and problems in China. J Subst Abus. 2000;5:71-8.

58. Xinhua News Agency. China to remain world's largest beer producer. Global Times. 200924 August.

Ready to submit your research? Choose BMC and benefit from:

- fast, convenient online submission

- thorough peer review by experienced researchers in your field

- rapid publication on acceptance

- support for research data, including large and complex data types

- gold Open Access which fosters wider collaboration and increased citations

- maximum visibility for your research: over $100 \mathrm{M}$ website views per year

At $\mathrm{BMC}$, research is always in progress.

Learn more biomedcentral.com/submissions 